\title{
Mass-Analyzed Threshold Ionization (MATI) Spectroscopy of Atoms and Molecules using VUV Synchrotron Radiation
}

\author{
Oleg Kostko, Sang Kyu Kim, ${ }^{\text {a) }}$ Stephen R. Leone, ${ }^{1}$ and Musahid Ahmed.* \\ Chemical Sciences Division, Lawrence Berkeley National Laboratory, Berkeley, CA 94720, \\ USA \\ ${ }^{1}$ Department of Chemistry and Physics, University of California at Berkeley, Berkeley, CA \\ 94720, USA \\ a) on sabbatical leave from KAIST, Korea \\ * Corresponding author: MS: 6R-2100, Lawrence Berkeley National Laboratory, 1 Cyclotron Road, \\ Berkeley, CA-94720, USA. Phone: (510) 486-6355; fax: (510) 486-5311; e-mail: MAhmed@lbl.gov
}

\begin{abstract}
Mass-analyzed threshold ionization (MATI) spectroscopy using synchrotron radiation (Advanced Light Source, Lawrence Berkeley National Laboratory) has been performed for $\mathrm{Ar}, \mathrm{N}_{2}, \mathrm{O}_{2}, \mathrm{~N}_{2} \mathrm{O}$, $\mathrm{H}_{2} \mathrm{O}, \mathrm{C}_{2} \mathrm{H}_{2}$, and $\mathrm{C}_{6} \mathrm{H}_{6}$. MATI allows for a better determination of ionization energies compared to those derived from photoionization efficiency curves traditionally used in synchrotron photoionization mass spectrometry. The separation of the long-lived Rydberg state from the directly-formed prompt ion, essential for a meaningful MATI spectrum, has been accomplished by employing an arrangement of ion optics coupled to unique electric-field pulsing schemes. For Ar, a number of resolved bands below the ionization energy are observed, and these are ascribed to high-n,I Rydberg states prepared in the MATI scheme. The first vibrational state resolved MATI spectra of $\mathrm{N}_{2}$ and $\mathrm{O}_{2}$ are reported and spectral characteristics are discussed in comparison with previously-reported threshold photoelectron spectroscopic studies. While MATI performed with synchrotron radiation is intrinsically less sensitive compared to laser based sources, this work demonstrates that MATI spectroscopy performed with widely tunable VUV radiation is a complementary technique for studying the ionization spectroscopy of polyatomic molecules.
\end{abstract}




\section{Introduction}

Photoionization spectroscopy provides information on the energetics and structures involved in the removal of a valence electron in a chemical system. High energy resolution, from the spectroscopic point of view, is critical since it provides the precise values of the ionization energies and rovibrational frequencies, which allows elucidation of the molecular structure of cations. In this respect, the zero-electron kinetic energy (ZEKE) ${ }^{1,2}$ and the mass-analyzed threshold ionization (MATI) techniques ${ }^{3}$ are considered to be the most highly-resolved ionization spectroscopic tools at the present time. Both techniques rely on the delayed pulsedfield ionization (PFI) of the long-lived Rydberg states of atoms or molecules, providing an energy resolution of less than $1 \mathrm{~cm}^{-1}$. The only difference between ZEKE and MATI spectroscopic methods is whether the electron or ion is detected, respectively, as the excitation energy is varied over the ionization thresholds. It is widely accepted now that stray fields present in most experimental conditions are responsible for the extensive l-type mixing among high-n Rydberg states, leading to the long-lived high-l, $m_{l}$ states which are then subjected to pulsed-field ionization ${ }^{4}$ providing ZEKE or MATI signals.

In the preceding two decades, ZEKE or MATI studies via either the $\left(1+1^{\prime}\right)$ two photon or one-photon vacuum ultraviolet (VUV) excitations have been extensively investigated for a number of chemical systems. ${ }^{2,5,6}$ These studies provide not only accurate and precise values of ionization energies but also those of cationic rovibrational frequencies from which the role of the particular molecular orbital in the structural layout of the molecule can be inferred. Even though ZEKE and MATI provide basically the same information, it seems that the former is more appropriate for rovibrational spectroscopic studies because of its higher resolving power, whereas the latter turns out to be quite useful in the dynamic study of clusters, conformers, and excited-state cations due to its mass-identifying ability. As a matter of fact, in MATI spectroscopy, one can not only identify the species responsible for the ionization but also separate and isolate the unique chemical species confined at a specific quantum state or in a specific conformational structure. Recently, a number of photoionization studies using the MATI technique for the selection of a specific size of the cluster, ${ }^{7}$ a specific conformational isomer, ${ }^{8,9}$ or a specific cationic quantum state ${ }^{10,11}$ have been reported.

One shortcoming of laboratory-based ZEKE/MATI spectroscopy lies in the lack of the VUV wavelength tunability as the ionizing radiation source. The $\left(1+1^{\prime}\right)$ two-photon excitation 
scheme requires the existence of stable intermediate states, and it is thus not applicable to molecular systems that have short-lived electronically excited states. VUV generation via fourwave mixing or tripling is efficient, however a number of different gases such as $\mathrm{Xe}, \mathrm{Kr}, \mathrm{Ar}, \mathrm{Hg}$, or $\mathrm{Mg}$ are required to cover the wide wavelength needed for ionization. The ionization energies of chemical systems are mostly in the range of $7-15 \mathrm{eV}$, and it would be ideal if one could generate a narrow band-width VUV radiation source which is widely tunable. In this respect, tunable synchrotron radiation coupled to a monochromator provides the ideal VUV source for molecular ionization. Hence, photoionization efficiency (PIE) spectra using synchrotron VUV radiation sources have been extensively used for IE determination and identification of a number of chemical systems. Accordingly, the combination of a synchrotron radiation source and highly energy-resolving ZEKE or MATI techniques should provide the ideal tool for universal high-resolution photoionization spectroscopy.

While PFI photoelectron (PFI-PE) ${ }^{12}$ and $\mathrm{ZEKE}^{13}$ spectroscopy with synchrotron radiation had been successfully applied to several chemical systems, MATI spectroscopy with a synchrotron source, because of its relatively low sensitivity, has had limited success. The $\mathrm{Ng}$ group reported in 1996 the first synchrotron-MATI spectra of Ar and Ne using quadrupole mass spectrometry, ${ }^{14}$ and recently, a synchrotron-MATI spectroscopy study using time-of-flight mass spectrometry has been reported for $\mathrm{Ar}$ and $\mathrm{N}_{2} \cdot{ }^{15}$ It should be pointed out that previous synchrotron-MATI studies had been limited only to atomic or diatomic systems where the Rydberg state lifetimes are sufficiently long-lived to allow detection.

In this report, we present synchrotron-MATI spectra using reflectron time-of-flight mass spectrometry coupled to a simple but unique PFI scheme for the effective separation of the MATI signal from the prompt ion signal. It has been demonstrated here for the first time that synchrotron-MATI can be applied even to polyatomic molecules for which high- $n$ Rydberg states undergo a number of dephasing channels. As mentioned above, the MATI technique is quite useful in its application to further novel dynamic studies of state- or structure-selected cations. Therefore, the endeavor of this work is to develop a universal synchrotron-MATI technique, while challenging experimentally, promises a wider and deeper application of Rydberg state spectroscopy to ion chemistry and photoionization dynamics. 


\section{Experimental Section}

The experiment was performed on a time-of-flight (TOF) mass spectrometer coupled to a $3 \mathrm{~m}$ monochromator at the chemical dynamics beamline (9.0.2) of the Advanced Light Source. The experimental machine is a molecular beam apparatus recently described for investigation of laser ablated species ${ }^{16}$ and hydrogen bonded clusters. ${ }^{17,18}$ Samples used in this study were supersonically expanded through a $100 \mu \mathrm{m}$ diameter nozzle located inside the source chamber. The gaseous samples $\left(\mathrm{Ar}, \mathrm{N}_{2}, \mathrm{O}_{2}, \mathrm{C}_{2} \mathrm{H}_{2}\right.$, and $\mathrm{N}_{2} \mathrm{O}$ ) were used directly from a cylinder, while helium carrier gas was used for seeding water or benzene. The supersonic beam was skimmed through a $2 \mathrm{~mm}$ diameter skimmer and intersected by a VUV beam inside the interaction region of the TOF ion optics. Ion optic electrodes have dimensions of $76 \times 76 \mathrm{~mm}^{2}$. The accelerator plate has an opening with a diameter of $38 \mathrm{~mm}$ covered with fine mesh. The plates of the ion optics are 12.7 $\mathrm{mm}$ apart, shown in Fig. 1. During conventional photoionization efficiency (PIE) curve measurements, the tunable VUV radiation intersects the supersonic beam in the center of the space between the repeller and accelerator plates (shown as 1 in Fig. 1). For the MATI experiment, the interaction position of VUV light with respect to the molecular beam was shifted by $\sim 2 \mathrm{~cm}$ closer to the source region. This allows the separation of directly ionized prompt ions from Rydberg states during their flight to the center of the ion optics. To be able to change the position of the interaction region of the VUV radiation and molecular beam, an X-Y translation stage, which allows movement of the whole experimental chamber with $\mathrm{mm}$ precision with respect to the stationary VUV beam-line, was implemented.

Two different electric field pulsing schemes were used for the separation of Rydberg states from prompt ions. In scheme I, two electrodes E1 and E2 (Fig. 1) had almost equal potentials of 1200 and $1199.5 \mathrm{~V}$, respectively. A weak field of less than $1 \mathrm{~V} / \mathrm{cm}$ between E1 and E2 allowed the separation of the prompt ions from the neutral Rydberg-tagged species before pulsed field ionization, as shown in Figs. 1 and 2. Because the synchrotron VUV radiation is quasi-continuous $(500 \mathrm{MHz})$, the pulsed-field ionization of Rydberg-states takes place via the application of a $1.5 \mu$ s-long $150 \mathrm{~V}$-pulse added on to the static potential of $1200 \mathrm{~V}$ at E1 at a frequency of $15 \mathrm{kHz}$. This pulsed-field ionizes the Rydberg-tagged neutrals and the same time pushes both prompt and MATI ions toward E2 for further acceleration into the TOF mass spectrometer. The time-dependent electrical signal from the microchannel plate is collected by a multichannel-scalar card and thereafter integrated with a PC computer. 
In scheme II, whereas the repeller electrode E1 was being pulsed similar to scheme I, a pulsed potential was applied to electrode E2 1.8 $\mu$ s prior to the electric pulse at E1, Figs. 1 and 2. This initial electric pulse at E2 further facilitated the separation of the MATI ion signal from the prompt ion signal. Time-of-flight spectra were recorded over the photon energy range in the vicinity of ionization thresholds. Each spectrum was integrated for 10 to 60 seconds depending on sample. The typical photon energy step used in this study varied between $3-5 \mathrm{meV}$. The MATI spectra were obtained by integrating over the peaks in the mass spectrum collected at each photon energy. An argon gas filter was used to filter out higher order harmonics of the undulator radiation. The absorption spectra of argon in the gas filter recorded with a $\mathrm{Si}$ photodiode was used for energy calibration of the MATI spectra.

\section{Results and Discussion}

\section{Pulsing schemes}

The synchrotron radiation source is quasi-continuous (500 MHz, 70 ps duration), and it is not trivial to separate the pulsed-field ionized species from the prompt ions in the time-offlight mass spectrometer due to the persistent production of both prompt and MATI ions along the molecular beam direction. In laboratory-based laser MATI spectroscopy, since the typical pulse duration of the laser is about $5 \mathrm{~ns}$, ion bunches exist in a finite time scale and it is quite straightforward to separate out those ions in space by the application of an appropriate electric pulse. Continuous ionization by the synchrotron radiation, however, results in a large linear distribution of both directly ionized molecules and ionized Rydberg states toward the TOF detector when the ion optics are pulsed. A TOF profile found in a typical time-of-flight spectrum corresponding to $\mathrm{N}_{2}{ }^{+}$obtained with the pulse scheme I (vide supra) is shown in Fig. 2 (a). One can clearly distinguish two peaks; a broad one with a maximum intensity located at the flight time of $\sim 22.46 \mu \mathrm{s}$ and a sharp peak at $\sim 22.53 \mu \mathrm{s}$. The sharp peak is ascribed to the MATI ion signal, whereas the broad one turns out to be due to prompt ions. The prompt ions are broadened and shifted in the time-of-flight spectrum due to both a space-charge effect and the spread in space due to the small static spoil field given prior to the electric pulse for PFI, Fig. 1. Neutral high- $n$, I Rydberg states for which the flight paths are not affected by the spoil field are then responsible for the sharp peak in the TOF spectrum. The major disadvantage of this method is the partial overlapping of these two peaks. This could be partly overcome if the VUV 
light-molecular beam interaction region is shifted further towards the source chamber, in such a way that only well separated ions may be transferred into the TOF mass spectrometer.

In the pulse scheme II, a more distinct separation of MATI and prompt ions is accomplished. Namely, by the application of the electric pulse going down from 1200.5 to 1190 $\mathrm{V}$ on $\mathrm{E} 2$ at $\sim 1.8 \mu$ s prior to the electric pulse at E1 going up from 1200 to $1350 \mathrm{~V}$, an effective pulsed spoil field of $7.9 \mathrm{~V} / \mathrm{cm}$ is applied for a better separation of MATI and prompt ions, Figs. 1 and 2(b). It should be noted however that the spoil field on E2 also contributes to ionization of Rydberg species up to $\sim 4 \sqrt{7.9} \mathrm{~cm}^{-1}$ (corresponding to $1.4 \mathrm{meV}$ ) below the uppermost MATI states in the adiabatic limit. ${ }^{2,19}$ Therefore, the first ion packet which travels faster consists of the prompt ions and ionized Rydberg states due to the spoil field on E2 whereas the second ion packet of the pure MATI ions created by PFI at E1 arrives at the MCP detector at later times. The spoil field of $7.9 \mathrm{~V} / \mathrm{cm}$ for the duration of $1.8 \mu$ s prior to PFI is found to be very effective in separating those two ion bunches in the time-of-flight. A typical example of the TOF-profile which is obtained for acetylene by employing the pulse scheme II is shown in Figure 2 (b). A sharp peak with a maximum at $22.15 \mu$ s corresponds to the pure MATI signal whereas a broad one with the maximum at $\sim 22.04 \mu \mathrm{s}$ is due to the admixture of the prompt and PFI ion signals. In order to understand and improve the pulsing scheme, a theoretical simulation was performed with SIMION software (version 8). The spectrum obtained in this simulation is shown in Figure 2 (c). It is obvious that the broad feature appearing at the early arrival time results from two peaks, of which one is produced by directly ionized ions (shown as blue line in Fig. 2 (c)) and the other (shown in red in Fig. 2 (c)) originates from Rydberg states ionized by the spoil field of 7.9 $\mathrm{V} / \mathrm{cm}$. The sharp peak at the later time is entirely due to MATI ions and is well reproduced in the simulation, Fig. 2 (b) and (c).

\section{Ar MATI}

The synchrotron-MATI spectrum of Ar taken by pulse scheme I shows a strong peak at the energy corresponding to the ionization threshold leading to the ground cationic state $\left({ }^{2} \mathrm{P}_{3 / 2}\right)$, Fig. 3. For Ar, the pure MATI signal could be completely isolated from prompt ions even with the pulse scheme I by tilting the ion flight path around using deflection plates placed perpendicular to the time-of-flight axis. The ionization potential of Ar from this spectrum is determined to be $15.787 \mathrm{eV}$, and this is quite consistent with the literature value of $15.760 \mathrm{eV}$ measured by absorption spectroscopy. ${ }^{20}$ It should be noted however that the IE value reported here, due to 
the relatively broad spectral bandwidth of the synchrotron radiation source (6-10 meV), does not necessarily represent the more refined value compared to previously reported ones. Moreover, the latter value is used for an energy calibration and thereafter in the paper two values of the experimental IE will be presented, an actual value and one calibrated according to the $\operatorname{Ar}$ literature value of $15.760 \mathrm{eV}$, in brackets, e.g. for argon 15.787 (15.760) eV. The discrepancy between obtained and literature values could be partially explained by a backlash in the mechanical system rotating the grating in the monochromator. The value, corresponding to the mechanical error, lies within 6 meV. Together with the final width of the ionizing radiation and the photon energy step this leads to the typical measurement error of $0.019 \mathrm{eV}$. Though there is an error with the absolute energy scale, which could be overcome with an energy calibration, the relative scale between spectra is correct for each scan and hence spectroscopy is still possible.

An interesting feature in the Ar MATI spectrum is the existence of a number of small peaks below the ionization threshold. Considering the adiabatic ionization depth of several meV at the given PFI condition, a long series of peaks below the ionization potential is not expected just from the PFI mechanism. A possible scenario is that as Ar is excited to a high-n Rydberg state, it may experience collisions in the beam during the flight time of $5-20 \mu$ s before PFI, resulting in an energy exchange that can likely enhance ionization. In fact, it is interesting to note that the high $n$ Rydberg state has an effective radius $(r)$ that approximately increases with $n^{2}$. This leads the collision cross section $(\sigma)$ to be proportional to $n^{4}$ since $\sigma \propto r^{2}$. This simple energy dependence of the collision cross section seems to be consistent with the intensity pattern observed in the Ar MATI spectrum, Fig. 3. Possible Rydberg series responsible for such peaks would be the $n s, n s^{\prime}, n d$, and $n d^{\prime}$ series where $n$ represents the principal quantum number. Here, the $s$ and $d$ converge to the ground cationic ${ }^{2} P_{3 / 2}$ state whereas $s^{\prime}$ and $d^{\prime}$ converge to the ${ }^{2} \mathrm{P}_{1 / 2}$ state. The Rydberg state spectroscopy of these $s$ and $d$ series of Ar are very well documented, ${ }^{20}$ and yet the MATI spectrum in Fig. 3 cannot simply be explained by these $s\left(s^{\prime}\right)$ and $d\left(d^{\prime}\right)$ series. This fact could indicate that severe l-type coupling among Rydberg states may occur in the presence of the stray and/or spoil field in the current experimental condition. In other words, even though the initial optically prepared Rydberg series should belong to $n s, n s^{\prime}$, $n \mathrm{~d}$, or $n \mathrm{~d}^{\prime}$, the resultant MATI spectrum may reflect the higher-I Rydberg states prepared through state mixing, which cannot clearly be identified at the present time. This indicates that Rydberg states revealed in the MATI spectrum could be somewhat different from direct 
excitation such as observed in resonance-enhanced multiphoton ionization (REMPI). This phenomenon had previously been observed for pulsed-field ionization photoelectron (PFI-PE) spectroscopy of $\mathrm{N}_{2}{ }^{21}$ The long lifetime of Ar Rydberg states seems to ensure the l-type state mixing prior to the pulsed-field ionization. ${ }^{22}$ The absence of a MATI peak for the upper spin-orbit $\left({ }^{2} \mathrm{P}_{1 / 2}\right)$ state of Ar suggests that the lifetime of this Rydberg state, once it is in the ionization continuum, decreases quite sharply, which is consistent with previous observations. ${ }^{14,15}$

\section{$\mathrm{N}_{2}$ and $\mathrm{O}_{2} \mathrm{MATI}$}

For diatomic molecules, a synchrotron-MATI has been reported only for $\mathrm{N}_{2}$ recently by Zhang et al. ${ }^{15}$ They modified the ion optics in a TOF mass spectrometer such that ions directly formed by synchrotron radiation could be avoided in the region of PFI. However, the presented MATI spectrum of $\mathrm{N}_{2}{ }^{15}$ is found to have contributions from both directly-formed prompt or autoionized ion signals and MATI, implying that their ion optics arrangement was not optimal. In our arrangement using the pulse scheme II, the complete separation of MATI from the prompt ions is accomplished to give a pure $\mathrm{N}_{2}$ MATI spectrum in Fig. 4 (a). The ionization energy is determined here to be 15.649 (15.622) eV, which is consistent with the previously reported value of $15.582 \mathrm{eV}{ }^{23}$ The fundamental, first and second overtone bands of the N-N stretching mode of $\mathrm{N}_{2}{ }^{+}$are clearly observed in the MATI spectrum to give respective frequencies of 264, 527, and $780 \mathrm{meV}$. Interestingly enough, this work provides the first vibrationally resolved MATI spectrum of $\mathrm{N}_{2}$ although the MATI origin had been previously identified. ${ }^{15}$ In principle, the vibrational feature associated with the ${ }^{2} \Sigma_{\mathrm{g}}{ }^{+} \leftarrow{ }^{1} \Sigma_{\mathrm{g}}{ }^{+}$transition should reflect the structural change of $\mathrm{N}_{2}$ upon the ionization of the ground $\mathrm{N}_{2}$ into the ground $\mathrm{N}_{2}{ }^{+}$. In the MATI spectrum, the origin band is most strongly observed whereas the vibrational bands of $v=1-3$ have an order of magnitude smaller intensities. It is noteworthy that the vibrationally excited bands have more or less similar intensities to each other, Fig. 4 (a).

Photoelectron spectroscopy of $\mathrm{N}_{2}$ had been reported to be somewhat different from MATI, ${ }^{24}$ showing the strongest origin with a very rapid decay for the vibrationally excited bands. The PES spectrum had been reproduced very well by the Franck-Condon calculation, ${ }^{24,25}$ which is consistent with the small increase of the diatomic distance in the ${ }^{1} \Sigma_{\mathrm{g}}{ }^{+}$to ${ }^{2} \Sigma_{\mathrm{g}}^{+}$transition $(\sim 1.67 \%) .{ }^{26}$ The disagreement between PES and MATI spectra in terms of the vibrational intensity profile, therefore, suggests that the ionization efficiencies of $\mathrm{N}_{2}$ Rydberg states converging to each vibrational threshold of ${ }^{2} \Sigma_{\mathrm{g}}{ }^{+}$may vary in the MATI ionization scheme. From 
the zeroth order picture, this apparently indicates that the Rydberg state lifetime of $\mathrm{N}_{2}$ gets lengthened as it is associated with the more vibrationally excited levels of $\mathrm{N}_{2}{ }^{+}\left({ }^{2} \Sigma_{\mathrm{g}}{ }^{+}\right)$. A different but similar vibrational intensity feature had also been reported in the threshold photoelectron (TPE) spectroscopy of $\mathrm{N}_{2}$ associated with the ground $\left({ }^{2} \Sigma_{\mathrm{g}}{ }^{+}\right)$cationic state, ${ }^{27}$ showing the vibrational intensity profile in which the higher vibrationally excited states are more pronounced in terms of the spectral intensities. In the ionization of Rydberg states of $\mathrm{N}_{2}$, multichannel interactions are reported to be quite active among many bright and dark states converging to each rovibrational state in the ground and/or electronically excited state of the cation. ${ }^{28}$ Therefore, multichannel quantum defect theory (MQDT) ${ }^{29}$ should be employed for the further interpretation of detailed spectral intensity profiles, which is beyond the scope here at the present time.

The same experimental scheme has been applied to $\mathrm{O}_{2}$ to generate the first vibrationally resolved MATI spectrum, Fig. 4 (b). Since the bond distance becomes significantly shortened upon ionization, ${ }^{26}$ a long progression of the O-O stretching mode of $\mathrm{O}_{2}{ }^{+}$is observed in the MATI spectrum with a fundamental frequency of $223 \mathrm{meV}$ and an ionization energy of 12.097 (12.070) $\mathrm{eV}$. The latter value is in good agreement with the literature value of $12.070 \mathrm{eV}^{30}$ The vibrational intensity pattern is found to be very similar to that of the PFI-PE spectrum. ${ }^{31}$ For low vibrational bands up to $v=3$, lower $\left({ }^{2} \Pi_{1 / 2 g}\right)$ and upper $\left({ }^{2} \Pi_{3 / 2 g}\right)$ spin-orbit states are quite well separated in the spectrum. Band assignments are given in Fig. 4.

\section{MATI of polyatomic molecules}

As the number of atoms increases in polyatomic molecules, the number of dephasing pathways of the highly excited Rydberg state increases, and this generally leads to a shortening of the Rydberg state lifetime. Therefore, the MATI spectroscopy of polyatomic molecules is generally considered to be more challenging compared to that of an atom or diatomic molecules in terms of the spectral sensitivity. It is especially true for synchrotron-MATI spectroscopy because of the relatively low photon flux of about $10^{14}$ photons $/ \mathrm{s}$ in the quasi-continuous synchrotron radiation source. In this regard, the complete separation of the MATI signal from the relatively much stronger prompt ion signal is essential for polyatomic systems. In this work, we have obtained the synchrotron-MATI spectra of $\mathrm{C}_{2} \mathrm{H}_{2}, \mathrm{~N}_{2} \mathrm{O}, \mathrm{C}_{6} \mathrm{H}_{6}$, and $\mathrm{H}_{2} \mathrm{O}$ for the first time, Fig. 5. Given the preliminary nature of the synchrotron MATI spectroscopy of polyatomic molecules, only a brief description of the observations is provided. The $\mathrm{C}_{2} \mathrm{H}_{2}$ MATI spectrum 
shows the origin at 11.449 (11.422) eV whereas the fundamental and first overtone $v_{2}$ bands are found at 11.668 and $11.886 \mathrm{eV}$, respectively. The $\mathrm{v}_{4}$ band is also weakly observed at $11.533 \mathrm{eV}$, and this is consistent with a previously reported photoelectron spectrum. ${ }^{32}$ For $\mathrm{N}_{2} \mathrm{O}$, two spinorbit states $\left({ }^{2} \Pi_{3 / 2},{ }^{2} \Pi_{1 / 2}\right)$ of the cation are revealed clearly in the MATI spectrum for the $0-0^{+}$ transition at $12.907(12.880) \mathrm{eV}$ with the additional fundamental vibrational band at $\mathrm{v}_{1}=0.136$ eV. ${ }^{33,34}$ A PIE curve for $\mathrm{N}_{2} \mathrm{O}$ obtained simultaneously with the MATI spectrum shows the smoothly-rising onset corresponding to the MATI origin peak, Figure 5 (b), demonstrating that the synchrotron-MATI technique could be very useful in the determination of accurate ionization energies. The MATI spectrum of $\mathrm{C}_{6} \mathrm{H}_{6}$ and $\mathrm{H}_{2} \mathrm{O}$ shows strong origins at 9.275 (9.248) and 12.664 (12.637) eV, respectively, and these agree very well with previously reported IE values of $9.244 \mathrm{eV}$ for benzene ${ }^{35}$ and $12.617 \mathrm{eV}$ for $\mathrm{H}_{2} \mathrm{O},{ }^{36}$ confirming applicability of the energy calibration which is done with the $\mathrm{Ar} 0-0^{+}$transition (vide supra). Here we should point out that the experimental error cited above is unable to explain the difference in IE values obtained in this work from those already published, indicating an unknown source of experimental error, the search of which is underway.

The successful synchrotron-MATI spectra of these polyatomic molecules demonstrate that the MATI spectroscopy can be conventionally implemented at a synchrotron facility to provide a universal ionization technique that could be applied to a wide variety of chemical systems including biological molecules, hydrogen bonded, carbon and metal clusters, and radicals formed in combustion processes. There is room for improvement of the synchrotronMATI spectroscopic technique. These may include the use of a slit jet or counterpropagating VUV and molecular beams to increase the interaction volume of the molecule with synchrotron radiation and/or chopping of the quasi-CW synchrotron radiation source for a more efficient separation of prompt and MATI ions.

\section{Conclusions}

Mass-analyzed threshold ionization (MATI) spectroscopy combined with synchrotron radiation (Advanced Light Source, Lawrence Berkeley National Laboratory) as the ionization source has been presented in this paper. This ionization technique is universal as the synchrotron radiation source is widely tunable in the vacuum ultraviolet region for the primary ionization of chemical systems. Because of the quasi-continuous nature of the synchrotron source, application of synchrotron-MATI had previously been limited to simple systems such as 
Ar or $\mathrm{N}_{2}$. Here, we have employed an arrangement of ion optics coupled with unique pulsed schemes to effectively separate the MATI ion signal from the prompt ion signal to provide the first synchrotron MATI spectra of polyatomic molecules such as $\mathrm{N}_{2} \mathrm{O}, \mathrm{H}_{2} \mathrm{O}, \mathrm{C}_{2} \mathrm{H}_{2}$, and $\mathrm{C}_{6} \mathrm{H}_{6}$, promising its wide application to a variety of chemical systems.

\section{Acknowledgements}

This work was supported by the Director, Office of Energy Research, Office of Basic Energy Sciences, and Chemical Sciences Division of the U.S. Department of Energy under contract No. DE-AC02-05CH11231. S.K.K. wishes to thank the SBS foundation and was partly supported by a MURI program (SRL) from the Air Force Office of Scientific Research, contract No. FA9550-04-1-0242. Leonid Belau and Lynelle Takahashi are acknowledged for their help during the initial attempts to implement MATI at the synchrotron. 


\section{References}

(1) Mullerdethlefs, K.; Sander, M.; Schlag, E. W. Chem. Phys. Lett. 1984, 112, 291.

(2) Schlag, E. W. ZEKE spectroscopy; Cambridge Univeristy Press: Cambridge, UK, 1998.

(3) Zhu, L. C.; Johnson, P. J. Chem. Phys. 1991, 94, 5769.

(4) Merkt, F. Annu. Rev. Phys. Chem. 1997, 48, 675.

(5) Johnson, P. M. in Photoionization and Photodetachment; Ng, C. Y., Ed.; World Scientific: Singapore, 2000; pp.296-346.

(6) Softley, T. P. Int. Rev. Phys, Chem. 2004, 23, 1.

(7) Choi, K. W.; Kim, S. K.; Ahn, D. S.; Lee, S. J. Phys. Chem. A 2004, 108, 11292.

(8) Park, S. T.; Kim, S. K.; Kim, M. S. Nature 2002, 415, 306.

(9) Choi, S.; Kang, T. Y.; Choi, K. W.; Han, S.; Ahn, D. S.; Baek, S. J.; Kim, S. K. J. Phys. Chem. A 2008, 112, 7191.

(10) Lee, M.; Kim, M. S. J. Chem. Phys. 2007, 126, 154317.

(11) Woo, H. K.; Wang, P.; Lau, K. C.; Xing, X.; Chang, C.; Ng, C. Y. J. Chem. Phys. 2003, 119, 9333.

(12) Hsu, C. W.; Evans, M.; Ng, C. Y.; Heimann, P. Rev. Sci. Instrum. 1997, 68, 1694.

(13) Mullerdethlefs, K.; Schlag, E. W. Annu. Rev. Phys. Chem. 1991, 42, 109.

(14) Hsu, C. W.; Lu, K. T.; Evans, M.; Chen, Y. J.; Ng, C. Y.; Heimann, P. J. Chem. Phys. 1996, 105, 3950.

(15) Zhang, S.; Wang, Y. M.; Cao, Z. Z.; Zhang, B.; Wang, S. S.; Kong, R. H.; Zhao, Y. J.; Shan, X. B.; Sheng, L. S. Rev. Sci. Instrum. 2007, 78, 043104.

(16) Nicolas, C.; Shu, J. N.; Peterka, D. S.; Hochlaf, M.; Poisson, L.; Leone, S. R.; Ahmed, M. J. Am. Chem. Soc. 2006, 128, 220.

(17) Belau, L.; Wilson, K. R.; Leone, S. R.; Ahmed, M. J. Phys. Chem. A 2007, 111, 10075.

(18) Kostko, O.; Belau, L.; Wilson, K. R.; Ahmed, M. J. Phys. Chem. A 2008, 112, 9555.

(19) Unterberg, C.; Gerlach, A.; Jansen, A.; Gerhards, M. Chem. Phys. 2004, 304, 237.

(20) Yoshino, K. J. Opt. Soc. Am. 1970, 60, 1220.

(21) Merkt, F.; Softley, T. P. Phys. Rev. A 1992, 46, 302. 
(22) Merkt, F. J. Chem. Phys. 1994, 100, 2623.

(23) Morioka, Y.; Lu, Y.; Matsui, T.; Tanaka, T.; Yoshii, H.; Hayaishi, T.; Hall, R. I. J. Chem. Phys. 1996, 104, 9357.

(24) Gardner, J. L.; Samson, J. A. R. J. Chem. Phys. 1974, 60, 3711.

(25) Jain, D. C.; Sahni, R. C. Int. J. Quantum Chem. 1968, 2, 325.

(26) Huber, K. P.; Herzberg, G.; Linstrom, P. J., Mallard, W. G., Eds.; National Institute of Standards and Technology: Gaithersburg MD, 20899, http://webbook.nist.gov.

(27) Peatman, W. B.; Gotchev, B.; Gurtler, P.; Koch, E. E.; Saile, V. J. Chem. Phys. 1978, 69, 2089.

(28) Merkt, F.; Guyon, P. M. J. Chem. Phys. 1993, 99, 3400.

(29) Jungen, C.; Dill, D. J. Chem. Phys. 1980, 73, 3338.

(30) Tonkyn, R. G.; Winniczek, J. W.; White, M. G. Chem. Phys. Lett. 1989, 164, 137.

(31) Song, Y.; Evans, M.; Ng, C. Y.; Hsu, C. W.; Jarvis, G. K. J. Chem. Phys. 1999, 111, 1905.

(32) Reutt, J. E.; Wang, L. S.; Pollard, J. E.; Trevor, D. J.; Lee, Y. T.; Shirley, D. A. J. Chem. Phys. 1986, 84, 3022.

(33) Wiedmann, R. T.; Grant, E. R.; Tonkyn, R. G.; White, M. G. J. Chem. Phys. 1991, 95, 746.

(34) Frey, R.; Gotchev, B.; Peatman, W. B.; Pollak, H.; Schlag, E. W. Chem. Phys. Lett. 1978, 54, 411.

(35) Neuhauser, R. G.; Siglow, K.; Neusser, H. J. J. Chem. Phys. 1997, 106, 896.

(36) Tonkyn, R. G.; Wiedmann, R.; Grant, E. R.; White, M. G. J. Chem. Phys. 1991, 95, 7033. 


\section{Figure Captions:}

Figure 1: Scheme of the experimental setup. The interaction point of VUV light with the molecular beam in typical photoionization efficiency curve measurements is denoted by 1 . Trajectories of directly ionized ions and excited Rydberg states in the spoiling field of electrodes $\mathrm{E} 1$ and E2 are shown by blue and red lines, respectively, and denoted by 2 and 3, accordingly. Trajectories of the ions after pulsed field ionization of Rydberg states and prompt ions are shown by wide red and blue arrows, respectively. Though all ions are pushed towards the detector, only ions passing through an aperture in the electrode E3 are shown by arrows.

Figure 2: Time-of-flight spectra for: a) $\mathrm{N}_{2}$ measured using experimental scheme I; b) $\mathrm{C}_{2} \mathrm{H}_{2}$ measured using scheme II; and c) SIMION simulated spectrum of $\mathrm{C}_{2} \mathrm{H}_{2}$. Two peaks, one shown in red and one maximized at $22.14 \mu \mathrm{s}$ in c), are due to MATI ions; the broad feature shown in blue corresponds to directly ionized $\mathrm{C}_{2} \mathrm{H}_{2}$ signal; additionally the standard deviation of five simulation runs is shown in light gray color. Pulse sequences for the electrodes E1 and E2 are shown by blue lines for the experimental scheme I (a) and scheme II (b).

Figure 3: Synchrotron-MATI spectrum of Ar. Inset represents a scaled up region of the MATI spectrum in the range of 15.37-15.75 eV. Red lines represent the approximate collision cross section of the Rydberg atom (see the text). Rydberg series of $n s, n d, n s^{\prime}$, and $n d^{\prime}$ are shown in the inset for comparison. For this and the following figures in the paper, open circles represent the experimental data, the solid line is shown as a guide to the eye and is obtained by smoothing the experimental data.

Figure 4: Synchrotron-MATI spectra of (a) $\mathrm{N}_{2}$ and (b) $\mathrm{O}_{2}$. The positions for the $\mathrm{v}^{+}=0-3\left(\right.$ for $\left.\mathrm{N}_{2}\right)$ and two spin-orbit states of $\mathrm{v}^{+}=0-5\left(\right.$ for $\left.\mathrm{O}_{2}\right)$ levels are marked. For $\mathrm{O}_{2}$, the spectrum between 12.0 and $12.6 \mathrm{eV}$ is scaled up $\times 15$ and shown in red. The origin of the shoulder appearing in the spectrum of $\mathrm{N}_{2}$ at $15.69 \mathrm{eV}$ is currently not known. Additional experiments clarifying the origin of the shoulder will be undertaken.

Figure 5: Synchrotron-MATI spectra of (a) $\mathrm{C}_{2} \mathrm{H}_{2}$, (b) $\mathrm{N}_{2} \mathrm{O}$, (c) $\mathrm{C}_{6} \mathrm{H}_{6}$, and (d) $\mathrm{H}_{2} \mathrm{O}$. Positions of vibration levels for $\mathrm{C}_{2} \mathrm{H}_{2}$ and $\mathrm{N}_{2} \mathrm{O}$ are shown. For nitrous oxide the PIE curve is shown in red. IE's for $\mathrm{C}_{6} \mathrm{H}_{6}$ and $\mathrm{H}_{2} \mathrm{O}$ are shown as arrows. 


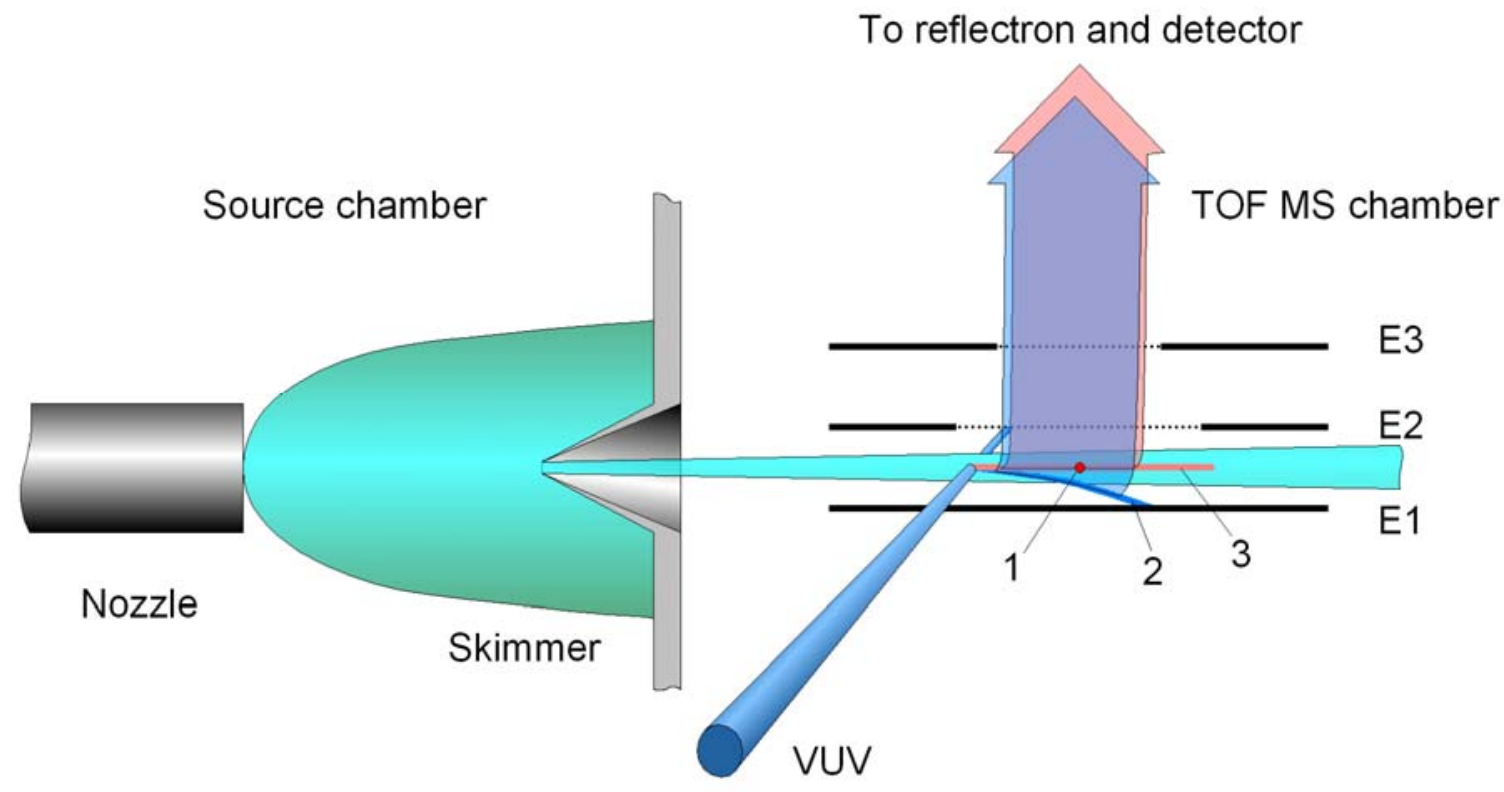

Figure 1. 

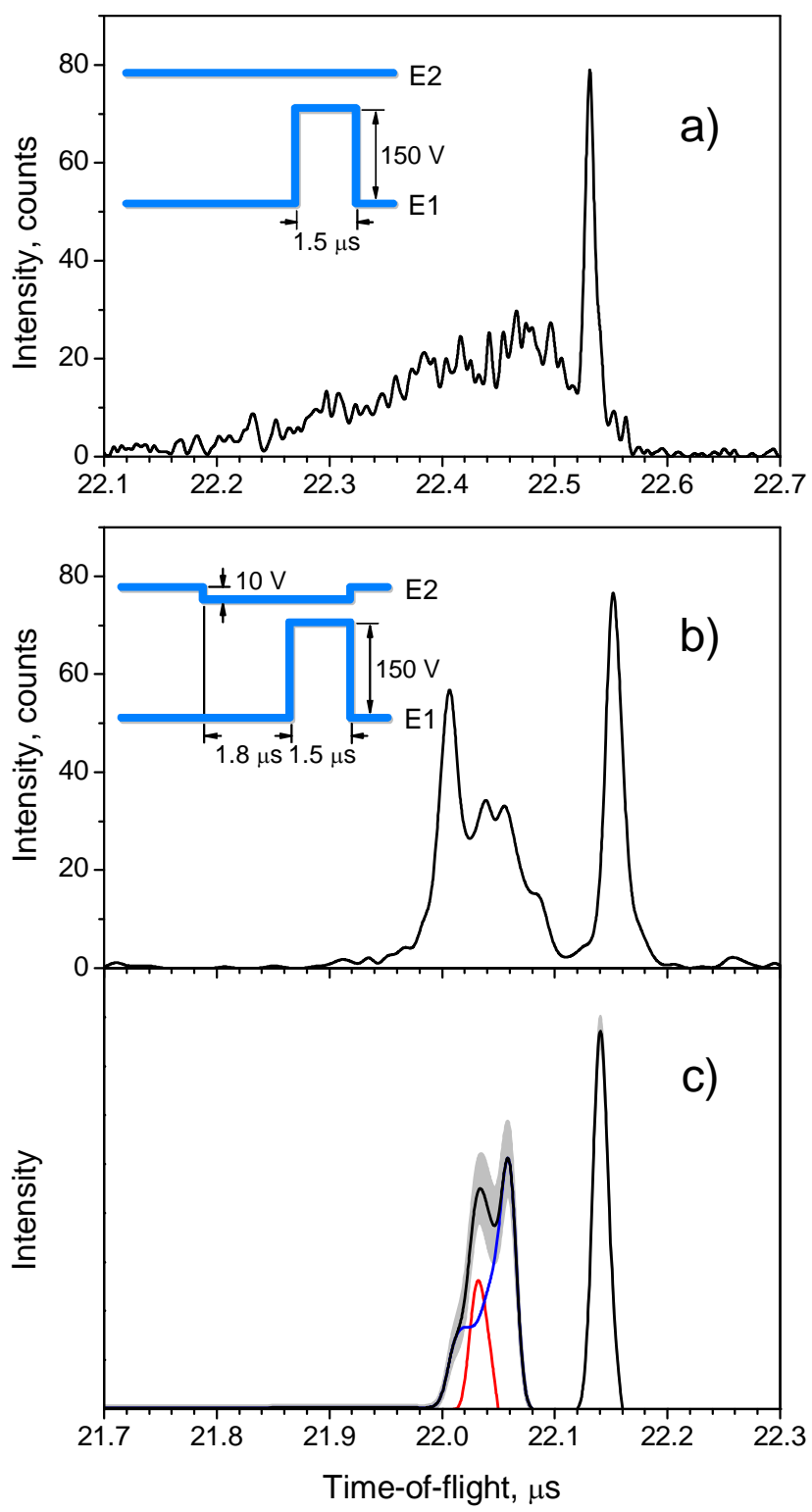

Figure 2. 


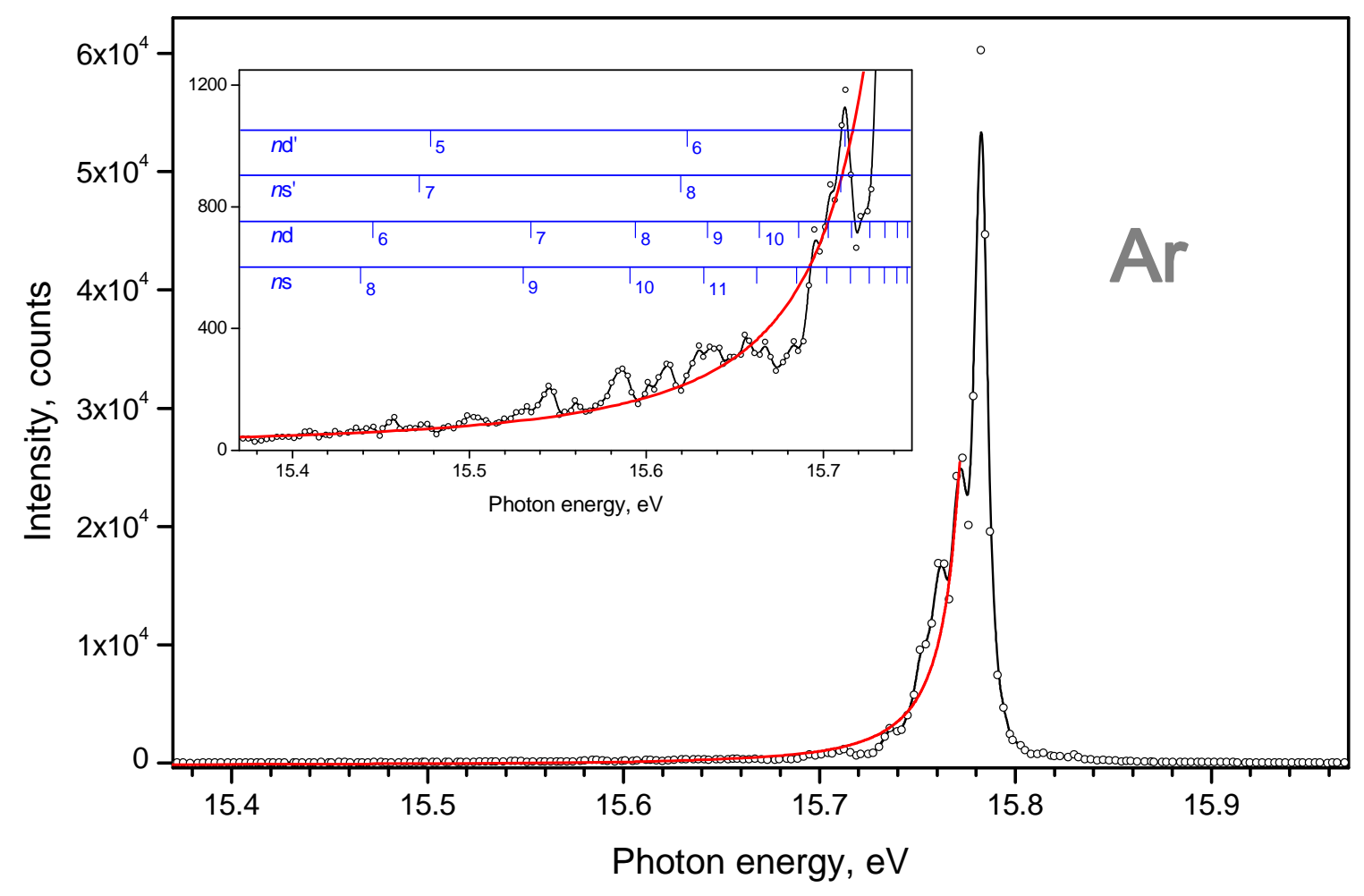

Figure 3. 

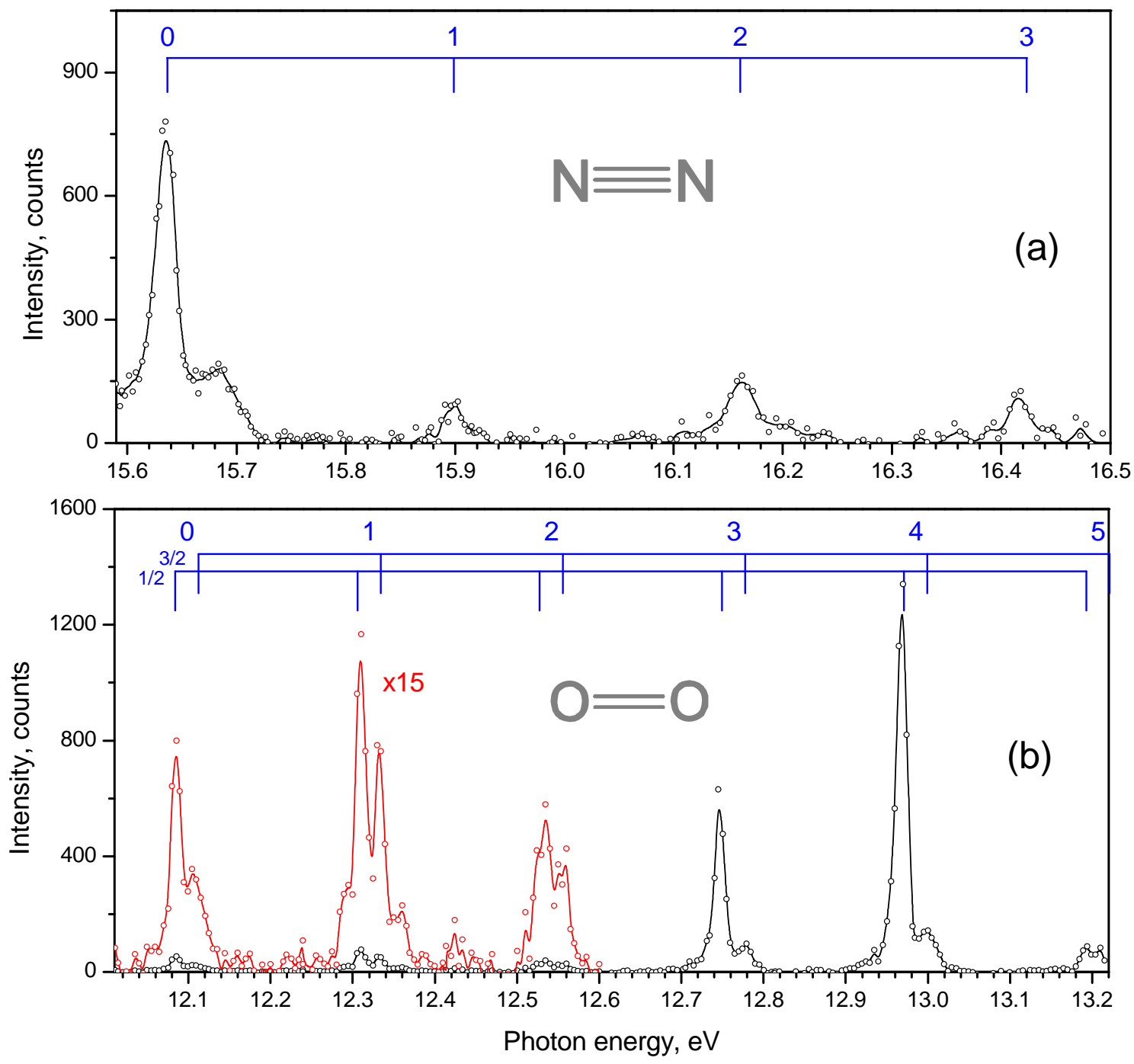

Figure 4. 

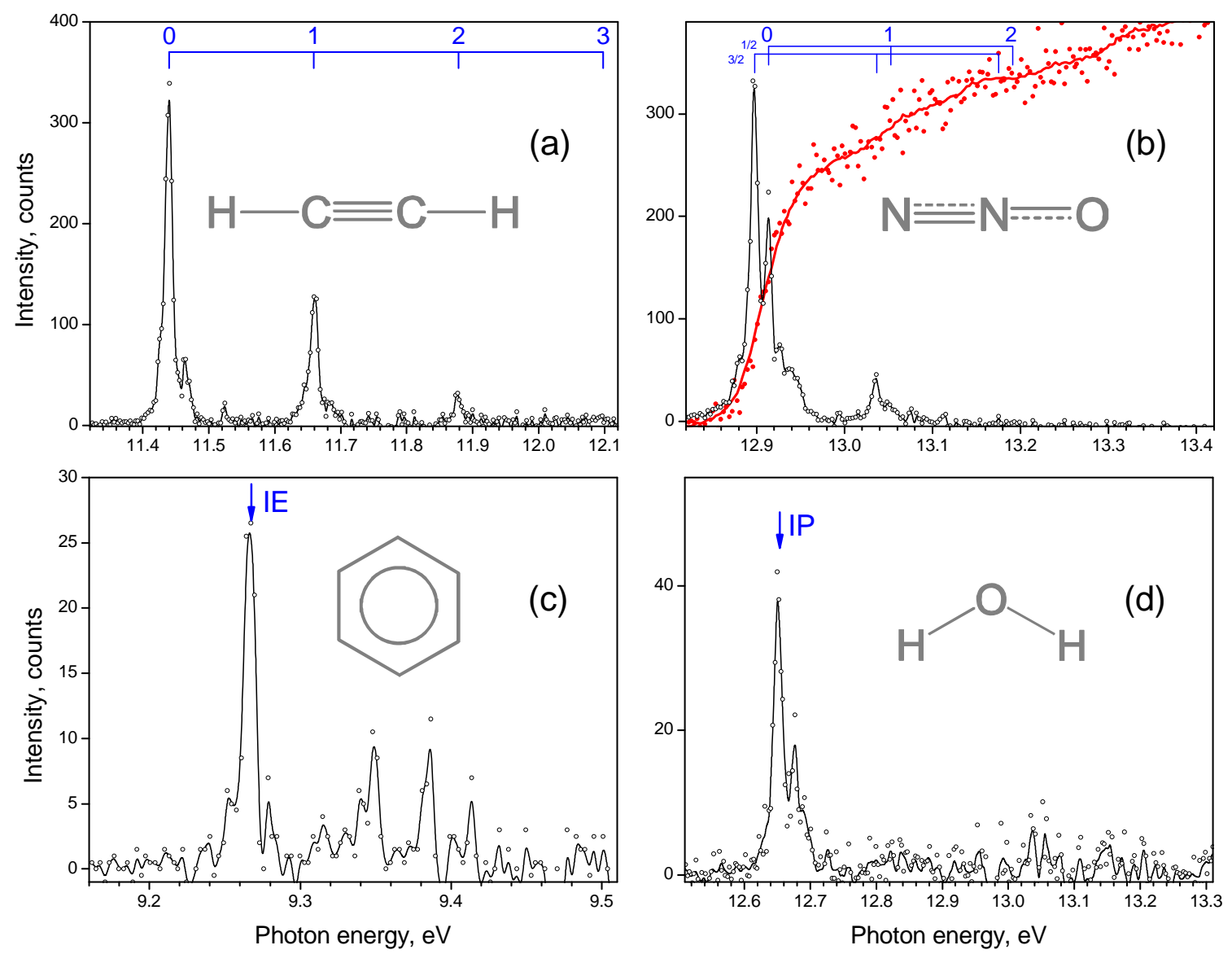

Figure 5. 